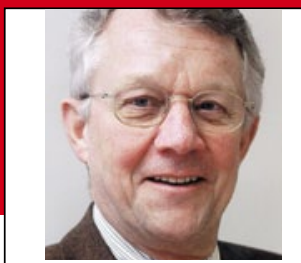

Prof. Dr. med. H.H olzgreve

Internist, Kardiologische Praxis, München

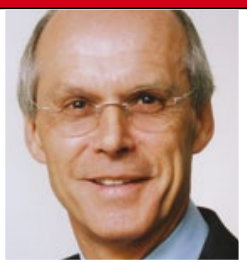

Prof. Dr. med. H. S. FüeßI

Isar-AmperKlinikum, KI. München-Ost, Haar

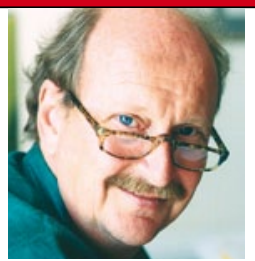

Prof. Dr. med.

E. Ernst

Peninsular

Medical School,

University

of Exeter/UK

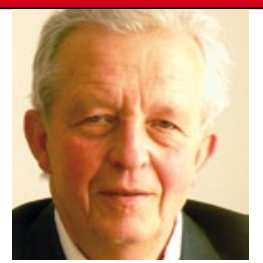

Prof. Dr. med. K. Malberg

Immunologie, DresdenLoschwitz

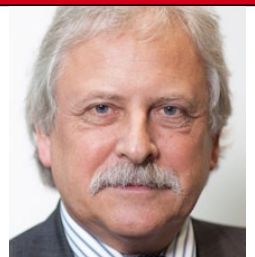

Prof. Dr. med. H.-C. Diener

Klinik für Neurologie, Universitätsklinikum Essen

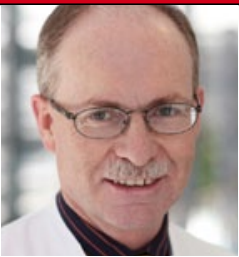

Prof. Dr. med. R. Büchsel

DRK Kliniken Berlin, Westend Klinik für Innere Medizin

\title{
D-Dimer bei Phlebothrombose: besser mit altersspezifischen Normalwerten
}

Weil die D-Dimer-Konzentrationen mit dem Alter ansteigen, können tiefe Venenthrombosen bei älteren Patienten auch bei höheren Grenzwerten sicher ausgeschlossen werden.

- Für die Studie rekrutierten 110 niedergelassene Ärzte in Holland 1374 konsekutive Patienten, bei denen aufgrund von Schwellung, Rötung und Schmerzen der unteren Extremität der Verdacht auf eine tiefe Venenthrombose bestand. Die klinische Wahrscheinlichkeit einer Thrombose wurde nach dem Wells-Score errechnet. Zusätzlich wurde das D-Dimer bestimmt. Nachweis bzw. Ausschluss einer Thrombose erfolgten durch eine Ultraschalluntersuchung am gleichen sowie am siebten Tag durch Spezialisten in Kliniken, die die Anamnesen und Befunde der Patienten nicht kannten.

Der zuverlässige Ausschluss einer Venenthrombose gelang bei altersspezifischen D-Dimer-Werten ab dem 50 . Lebensjahr signifikant häufiger als bei den üblichen Werten (47,8 vs. $42,0 \%$ ). Der übliche Grenzwert liegt bei $500 \mu \mathrm{g} / \mathrm{l}$. Bei der altersspezifischen Grenzwertbestimmung wird der Wert pro Lebensjahr über 50 um $10 \mu \mathrm{g} / \mathrm{l}$ angehoben. Die Tref- ferrate steigt mit dem Lebensalter und betrug schließlich bei über 80 -Jährigen 35,5 vs. $21,0 \%$ (s. Tab). Gleiche Ergebnisse ergaben sich, wenn der Grenzwert ab dem 60. Lebensjahr auf $750 \mu \mathrm{g} / \mathrm{l}$ erhöht wurde. Die Rate falsch negativer Ergebnisse änderte sich nicht nennenswert.

\section{Kommentar}

Die potenziell lebensgefährliche tiefe Venenthrombose wird mit dem Alter immer häufiger. Das D-Dimer gilt in jedem Alter als wichtiger diagnostischer Suchtest, doch steigen seine Werte mit dem Alter an, ohne dass bisher altersspezifische Normwerte definiert oder berücksichtigt wurden. Die vorliegende Untersuchung zeigt, dass die diagnostische
Zuverlässigkeit der D-Dimer-Bestimmung unter Praxisbedingungen deutlich ansteigt, wenn altersspezifische Normalwerte für das D-Dimer (entweder Alter in Jahren x 10 $\mu \mathrm{g} / \mathrm{l} \mathrm{ab}$ dem 50. LJ., also z. B. $650 \mu \mathrm{g} / \mathrm{l}$ bei 65-Jährigen oder generell $750 \mu \mathrm{g} / \mathrm{l} \mathrm{ab} \mathrm{dem}$ 60. Lebensjahr) verwendet werden. Diese Ergebnisse bedeuten, dass der niedergelassene Arzt einen Patienten mit geringer klinischer Wahrscheinlich für eine Venethrombose und negativen, altersspezifisch höheren D-Dimer-Grenzwerten nicht mehr zur Sonografie schicken muss.

H. HOLZGREVE =

- H.J. Schouten et al.

Validation of two age dependent D-dimer cut-off values for exclusion of deep vein thrombosis in suspected elderly patients in primary care: retrospective, cross sectional, diagnostic analysis. Brit. Med. J. BMJ 2012;344:e2985

\section{Tabelle}

Üblicher Grenzwert (500 $\mu \mathrm{g} / \mathrm{l})$

\begin{tabular}{|l|l|l|}
\hline Ausschluss & 272 & $42,0 \%$ \\
\hline Falsch negativ & 2 & $0,3 \%$ \\
\hline Ausschluss Pat. $>80$ & 13 & $21,0 \%$ \\
\hline
\end{tabular}

Altersspezif. Grenzwert (Alter in Jahren x $10 \mu \mathrm{g} / \mathrm{l}$ ab dem 50. LJ.)

Ausschluss

\begin{tabular}{|l|l|}
\hline 309 & $47,8 \%$ \\
\hline 3 & $0,5 \%$ \\
\hline 22 & $35,5 \%$ \\
\hline
\end{tabular}

Falsch negativ

Ausschluss Pat $>80$
Ausschluss von Venenthrombosen bei 647 Patienten mit geringer klinischer Wahrscheinlichkeit durch übliche und altersspezifische D-Dimer-Grenzwerte. 